\title{
Characterizing abnormal behavior in a large population of zoo-housed chimpanzees: Prevalence and potential influencing factors
}

\author{
Sarah L Jacobson ${ }^{1}{ }^{\text {, Stephen R Ross }}{ }^{\text {Corresp., }}{ }^{1}$, Mollie A Bloomsmith ${ }^{2}$ \\ 1 Lester E. Fisher Center for the Study and Conservation of Apes, Lincoln Park Zoo, Chicago, Illinois, United States \\ 2 Animal Resources, Yerkes National Primate Research Center, Atlanta, Georgia, United States \\ Corresponding Author: Stephen R Ross \\ Email address: sross@lpzoo.org
}

Abnormal behaviors in captive animals are generally defined as behaviors that are atypical for the species and are often considered to be indicators of poor welfare. Although some abnormal behaviors have been empirically linked to conditions related to elevated stress and compromised welfare in primates, others have little or no evidence on which to base such a relationship. The objective of this study was to investigate a recent claim that abnormal behavior is endemic in the captive population by surveying a broad sample of chimpanzees (Pan troglodytes), while also considering factors associated with the origins of these behaviors. We surveyed animal care staff from 26 accredited zoos to assess the prevalence of abnormal behavior in a large sample of chimpanzees in the United States for which we had information on origin and rearing history. Our results demonstrated that $64 \%$ of this sample were reported to engage in some form of abnormal behavior in the past two years and $48 \%$ of chimpanzees engaged in abnormal behavior other than coprophagy. Logistic regression models were used to analyze the historical variables that best predicted the occurrence of all abnormal behavior, any abnormal behavior that was not coprophagy, and coprophagy. Rearing had opposing effects on the occurrence of coprophagy and the other abnormal behaviors such that mother-reared individuals were more likely to perform coprophagy, whereas non-mother-reared individuals were more likely to perform other abnormal behaviors. These results support the assertion that coprophagy may be classified separately when assessing abnormal behavior and the welfare of captive chimpanzees. This robust evaluation of the prevalence of abnormal behavior in our sample from the U.S. zoo population also demonstrates the importance of considering the contribution of historical variables to present behavior, in order to better understand the causes of these behaviors and any potential relationship to psychological wellbeing. 
3 Characterizing abnormal behavior in a large population of zoo-housed chimpanzees:

4 Prevalence and potential influencing factors

5 Sarah L. Jacobson ${ }^{1}$, Stephen R. Ross ${ }^{1}$, Mollie A. Bloomsmith ${ }^{2}$

$6{ }^{1}$ Lester E. Fisher Center for the Study and Conservation of Apes, Lincoln Park Zoo, Chicago,

7 IL, USA

$82^{2}$ Yerkes National Primate Research Center, Atlanta, GA, USA

9

10

11

12 Corresponding Author:

13 Stephen R. Ross ${ }^{1}$

142001 N Clark St, Chicago, IL, 60614, USA

15 Email address: sross@lpzoo.org 


\section{Abstract}

18 Abnormal behaviors in captive animals are generally defined as behaviors that are atypical for

19 the species and are often considered to be indicators of poor welfare. Although some abnormal

20 behaviors have been empirically linked to conditions related to elevated stress and compromised

21 welfare in primates, others have little or no evidence on which to base such a relationship. The

22 objective of this study was to investigate a recent claim that abnormal behavior is endemic in the

23 captive population by surveying a broad sample of chimpanzees (Pan troglodytes), while also

considering factors associated with the origins of these behaviors. We surveyed animal care staff

from 26 accredited zoos to assess the prevalence of abnormal behavior in a large sample of

chimpanzees in the United States for which we had information on origin and rearing history.

Our results demonstrated that $64 \%$ of this sample were reported to engage in some form of abnormal behavior in the past two years and $48 \%$ of chimpanzees engaged in abnormal behavior other than coprophagy. Logistic regression models were used to analyze the historical variables that best predicted the occurrence of all abnormal behavior, any abnormal behavior that was not coprophagy, and coprophagy. Rearing had opposing effects on the occurrence of coprophagy and the other abnormal behaviors such that mother-reared individuals were more likely to perform coprophagy, whereas non-mother-reared individuals were more likely to perform other abnormal behaviors. These results support the assertion that coprophagy may be classified separately when assessing abnormal behavior and the welfare of captive chimpanzees. This robust evaluation of the prevalence of abnormal behavior in our sample from the U.S. zoo population also demonstrates the importance of considering the contribution of historical variables to present behavior, in order to better understand the causes of these behaviors and any potential relationship to psychological wellbeing. 
40 Characterizing abnormal behavior in a large population of zoo-housed chimpanzees:

41 Prevalence and potential influencing factors

42 Sarah L. Jacobson, Stephen R. Ross, \& Mollie A. Bloomsmith

\section{Introduction}

Abnormal behaviors in primates have been generally defined as behaviors that are

atypical of the species and/or occur at different frequencies in captivity than in the wild (Erwin \&

Deni, 1979; Walsh, Bramblett, \& Alford, 1982). These behaviors can result from both proximate and past exposure to chronic aversive stimuli, including environments that limit the ability to perform species-typical behaviors, repeated stressful procedures such as sedations for clinical procedures (Lutz, Well, \& Novak, 2003), and/or atypical early social experiences such as reduced or absent maternal care (Brent, Lee, \& Eichberg, 1989; Kalcher et al., 2008; Freeman \& Ross, 2014). Other intrinsic factors such as sex, species, and animal temperament (Vandeleest, McCowan, \& Capitanio, 2011; Gottlieb, Capitanio, \& McCowan, 2013) can also influence the expression of some abnormal behaviors. In part because of the association with suboptimal social and physical environments, abnormal behaviors are often considered to be reliable indicators of psychological distress and as such, poor welfare (Mason, 1991; Garner, 2005). Studying the prevalence and persistence of these behaviors in captive environments is critical to better understanding the factors contributing to the wellbeing of captive primates. abnormal behavior can be quite challenging for several reasons. The first is a lack of consistency in the types and definitions of behaviors considered to be atypical. Walsh, Bramblett, and Alford (1982) developed one of the most widely-used ethograms of abnormal behaviors in chimpanzees 
64 (see Nash et al., 1999; Hook et al., 2002; Birkett \& Newton-Fisher, 2011). Some classifications

65 distinguish between behaviors that are pathological and harmful (e.g. self-injurious behavior)

66 and those that are less severe (e.g. repetitive motions) (Bayne \& Novak, 1998). Other

67 investigators have not made such a distinction, treating a variety of abnormal behaviors as

68 functionally equivalent in terms of impact on the animal (Birkett \& Newton-Fisher, 2011). A

69 second challenge relates to the interpretation of the context in which particular behaviors are

70 performed. In some cases, behaviors can occur in both a species-typical context as well as those

71 that may be more likely tied to an underlying distressful state. For example, some researchers

72 have recognized this difficulty and advised that caution be used when categorizing rocking as an

abnormal behavior because of the difficulties in distinguishing instances when the behavior is

74 communicative in chimpanzees (Fritz et al., 1992; Ross \& Bloomsmith, 2011). These types of

75 definitional incongruities can lead to difficulties interpreting and comparing results of abnormal

76 behavior studies.

77 Perhaps the broadest challenge related to the study of abnormal behavior is related to the

78 interpretation of behaviors and their potential underlying etiologies. Although some behaviors

79 have been empirically linked to conditions related to elevated stress and compromised welfare,

80 others have little or no evidence on which to base such a relationship. Many stereotypies are

81 commonly used as indicators of reduced wellbeing, but this relationship is convoluted due to the

82 complex mechanisms underlying stereotypic behaviors. Mason and Latham (2004) suggest that

83 some stereotypies can develop as coping mechanisms for animals and with repetition shift into

84 an automatic behavior that is not necessarily reflective of their current environment. Other

85 stereotypies may even demonstrate a degree of behavioral flexibility as animals attempt to satisfy

86 a motivation to perform a natural behavior pattern in a captive environment (Mason \& Latham, 
87 2004). Additionally, the relationship between stereotypy and self-injurious behavior is poorly

88 understood, as some self-directed stereotypies can cause physical injury while the same behavior can also occur without injury. The classification of depilation and regurgitation and reingestion as problematic behaviors is also debated (Baker \& Easley, 1996; Hosey \& Skyner, 2007). Novak et al. (2006) advocated for further study of the biological bases of these categories of behavior to determine whether they represent different manifestations of the same underlying mechanism. Clearly, a better understanding of the etiologies of those behaviors classified as abnormal will be critical to determining which can be used as reliable indicators of negative welfare in captive primates.

One of the most comprehensively studied factors influencing the development of abnormal behaviors is early social experience and in particular, the rearing history of captive primates. Early maternal separation in captive macaques and chimpanzees has repeatedly been shown to lead to stereotypies, self-injurious behavior, and incompetent social and reproductive behavior (Harlow \& Harlow, 1965; Rogers \& Davenport, 1969; Fritz et al., 1992; Nash et al., 1999; Martin, 2002). Stress physiology has also supported this connection, demonstrating a dysregulation of the hypothalamic-pituitary-adrenocortical axis in primates that are separated from their mothers early in life, along with elevated expression of abnormal behaviors (Feng et al., 2012). In contrast, some abnormal behaviors in chimpanzees, such as coprophagy, appear to be more prevalent in mother-reared individuals compared to those raised by humans (Nash et al., 1999; Bloomsmith et al., 2006). Although not all primates who have experienced maternal separation demonstrate behavioral abnormalities, their rearing history appears to be an important factor influencing abnormal behavior. 
The physical environment experienced by captive primates may also play a role in the

110 development of abnormal behaviors, as the absence of appropriate sensory and motor stimulation

111 can lead to stereotypies (Berkson, Mason, \& Saxon, 1963; Harlow \& Harlow, 1965). When

112 captive environments fail to provide adequate opportunities for natural behavior patterns, some

113 atypical behaviors such as rocking or pacing may develop as a form of self-stimulation (Walsh,

114 Bramblett, \& Alford, 1982). Further evidence for the importance of physical environments

115 comes from studies that demonstrate reductions in abnormal behaviors following a move to a

116 more natural or enriched environment (Pfeiffer \& Koebner, 1978; Brent, Lee, \& Eichberg, 1989;

117 Schapiro \& Bloomsmith, 1994; Novak et al., 1998; Ross et al., 2011). Given this broad support

118 for a link between a primate's current and past physical and social environments, it seems

119 imperative to consider a wide range of potential influencing factors when investigating the 120 prevalence of abnormal behaviors.

121 In this study, we surveyed animal care staff to assess the prevalence (proportion of

122 individuals who exhibited a behavior at least once in a two year period) of abnormal behaviors in 123 a broad sample of chimpanzees living in accredited zoological parks. We also evaluated potential

124 links between historical variables and abnormal behaviors. Although many primates exhibit

125 abnormal behavior, chimpanzees are a particularly relevant species with which to investigate

126 these phenomena. Chimpanzees are a socially and cognitively complex species and they perform

127 a variety of abnormal behaviors in a variety of settings (Nash et al., 1999; Birkett \& Newton-

128 Fisher, 2011). Indeed, the captive chimpanzee population in North America provides a unique

129 opportunity to investigate factors influencing the development of abnormal behavior, in part

130 because of the inherent range of rearing and housing conditions experienced by these individuals.

131 Chimpanzees living in accredited zoos come from a broad diversity of backgrounds including 
132 those born and raised in zoos, those born in the wild and imported (many years ago) to North

133 America, and those born in other captive settings such as research laboratories or those privately

134 owned as pets or performers, and later moved to zoos.

135 A recent study of abnormal behavior in chimpanzees attempted to quantify the prevalence

136 and diversity of abnormal behaviors in the zoo setting and concluded that "abnormal behaviour is

137 endemic in captivity" (Birkett \& Newton-Fisher, 2011, p. 5). The study included 40 chimpanzees

138 at only six institutions to arrive at this conclusion. Here, we use a broader sampling of the zoo-

139 housed chimpanzee population to assess the prevalence of these behaviors, surveying animal

140 staff at 26 accredited zoos to collect data on 165 chimpanzees. Furthermore, we used

141 information on their origin and rearing histories to investigate the factors influencing the

142 expression of abnormal behaviors. Our objective was to characterize these populations and

143 gather information to help influence future management practices.

145 Methods

146 Data Collection

147 To assess the prevalence of abnormal behavior of a large sample of chimpanzees housed

148 in accredited zoos in the United States, we surveyed animal care staff who were familiar with

149 those individuals. Using PMCTrack software (2016), we administered an online questionnaire to

150 all Institutional Representatives of the Chimpanzee Species Survival Plan (SSP). These

151 individuals included curators, managers and zookeepers who worked regularly with the

152 chimpanzees and were responsible for their care and management. Respondents were asked to

153 note, for each individual chimpanzee at their institution, which abnormal behaviors were 
154 displayed at least once in the previous two-year period. This is an inherently conservative 155 approach that is more likely to overestimate the prevalence of abnormal behaviors than to 156 underestimate it because even highly infrequent behaviors could be recorded. The list of

157 abnormal behavior categories (Table 1) was developed in part from Birkett and Newton-Fisher 158 (2011) to facilitate comparisons with the results of that study.

Table 1. The definitions of abnormal behavior categories for chimpanzees used in this study

\begin{tabular}{ll}
\hline Coprophagy & Ingestion of feces \\
\hline Hair Pluck & Pulling out hair on self or another \\
\hline Rock & $\begin{array}{l}\text { Repetitive and sustained swaying movement without } \\
\text { piloerection }\end{array}$ \\
\hline Regurgitation \& Reingestion & $\begin{array}{l}\text { The deliberate regurgitation of food and subsequent } \\
\text { consumption of the food }\end{array}$ \\
\hline Self-injurious Behavior & Biting, picking, or scratching at own body to cause injury \\
\hline Pacing & $\begin{array}{l}\text { Locomoting repetitively along the same path with no clear } \\
\text { objective }\end{array}$ \\
\hline Other & Any other behavior deemed abnormal, space to describe \\
\hline
\end{tabular}

Historical variables

Information about the chimpanzees, including their sex, rearing, and origin was drawn from the North American Regional Studbook for Chimpanzees (Ross, 2015). Rearing was simply categorized as mother-reared or non-mother-reared as many historical records did not provide sufficient information to be more precise about rearing conditions. The origin of individuals was defined as the location at which the chimpanzee was born, and therefore the environment where

168 it spent at least some of its early developmental period. Origins were categorized as zoo,

169 laboratory, wild, and private. The private designation included chimpanzees that were kept as 
170 pets or performers and therefore had significant human interaction during their development

171 (Freeman \& Ross, 2014).

172 Facilities and Subjects

173 We received surveys on 181 chimpanzees, but not all were complete. After removing

174 incomplete surveys and excluding chimpanzees with unknown historical variables, our study

175 sample consisted of 165 chimpanzees (see Table $2 \mathrm{a} \& 2 \mathrm{~b}$ for breakdown of independent

176 variables). Chimpanzees ranged in age from 2-78 years old and age category distributions were

177 comparable to those of the overall AZA population with $10 \%$ immature $(<11), 65 \%$ adult (11-

17840 ), and 25\% elderly (>40) (Ross, 2015). They were all socially housed at one of 26 zoos

179 accredited by the Association of Zoos and Aquariums (AZA). Though there was some variability

180 in physical environments and management practices, all individuals lived under the regulatory

181 framework provided by AZA and care was guided by the principles in the AZA Chimpanzee

182 Care Manual (AZA Ape Tag, 2010). The sample was 38\% male and 62\% female, which matches

183 the overall sex distribution of the entire AZA population (Ross, 2015).

184 Table 2a. Chimpanzees in study sample with each rearing history

\begin{tabular}{lcc}
\hline Rearing & Number of individuals & Percentage of sample \\
\hline Mother-reared & 107 & $65 \%$ \\
\hline Non-mother-reared & 58 & $35 \%$ \\
\hline
\end{tabular}

185

186 Table 2b. Chimpanzees in study sample from each origin category

\begin{tabular}{lcc}
\hline Origin & Number of individuals & Percentage of sample \\
\hline Laboratory & 15 & $9 \%$ \\
\hline Private & 15 & $9 \%$ \\
\hline Wild & 34 & $21 \%$ \\
\hline
\end{tabular}




\begin{tabular}{lll}
\hline Zoo & 101 & $61 \%$ \\
\hline
\end{tabular}

187

188 Analysis

189

We calculated the proportion of the sample who were reported to engage in any form of abnormal behavior, hereafter ABN-ALL. Additionally, due to the reported ambiguity of coprophagy as a reliable indicator of welfare (Nash et al., 1999; Hopper, Freeman, \& Ross, 2016), we calculated both the proportion of the sample that were reported to engage in coprophagy specifically (ABN-C), as well as the proportion who were reported to engage in any form of abnormal behavior except for coprophagy (ABN-XC). behavior was assessed using binary logistic regression modeling. The variable of sex was considered in this analysis due to inconsistent results evaluating its association with abnormal behavior in past studies (Fritz et al., 1992; Nash et al., 1999). Analyses were conducted in R (R Core Team, 2015). The reference variable for sex was female, for rearing was mother-reared, and for origin was wild. A separate model was run for each of the dependent variables: ABN-ALL, $\mathrm{ABN}-\mathrm{C}$ and $\mathrm{ABN}-\mathrm{XC}$. For all analyses an alpha value of $\mathrm{p} \leq 0.05$ was considered significant.

\section{Results}

Our survey indicated that $64 \%$ of the 165 chimpanzees had been seen to exhibit some form of abnormal behavior (ABN-ALL) at least once in the previous two years. Coprophagy

206 (ABN-C) was the most prevalent abnormal behavior, with $41 \%$ of chimpanzees reported to 207 engage in the behavior. Hair plucking was also fairly common, with $32 \%$ of the sample reported 
208 to engage in this behavior. Other behaviors were far less commonly reported (see Figure 1).

209 When removing chimpanzees that only exhibited coprophagy, $48 \%$ of the 165 chimpanzees

210 exhibited abnormal behavior (ABN-XC).

212 Figure 1. The percentage of the study sample reported to engage in each category of abnormal behavior at least once from 2011-2013

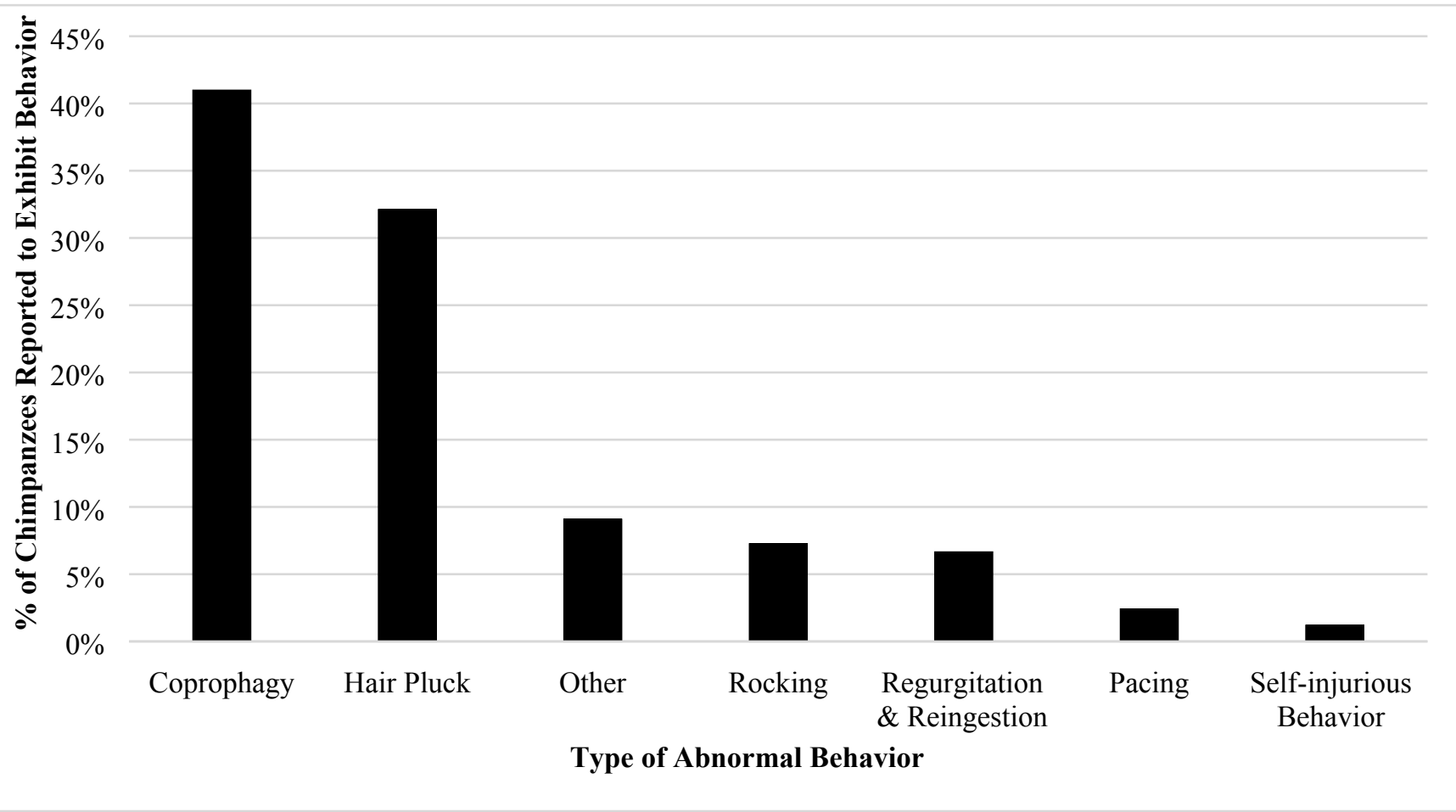

Logistic regression models were created using all combinations of the three historical of the dependent variables: ABN-ALL, ABN-C and ABN-XC. Only main effects were included in these models because all of the combinations of variables did not exist in our dataset, which compromised our ability to assess interaction effects. The best fit model was chosen through AIC comparison for each dependent variable (Symonds \& Moussalli, 2011). When multiple 
221 models were statistically equivalent, the model that included the most variables was chosen in

222 order to assess the influence of more factors on the chimpanzees' behavior. The best fit model

223 for ABN-ALL included sex and rearing (not origin) as predictor variables and was statistically

224 significant, $X^{2}(2)=6.49, p=0.04$. Table 3 reports the regression coefficients and the odds ratios

225 for the model. The model explained 5.3\% (Nagelkerke $\mathrm{R}^{2}$ ) of the variance in ABN-ALL and

226 correctly classified $61.8 \%$ of cases. The variable sex had a negative relationship with ABN-ALL

227 such that male chimpanzees were 2.04 times less likely to exhibit abnormal behavior than

228 females. Rearing was not a significant predictor of ABN-ALL in this model.

230 Table 3. Logistic regression model for any abnormal behavior (ABN-ALL) with predictor

231 variables and constant. Significant variables are bolded. $\mathrm{R}^{2}=0.03$ (Hosmer-Lemeshow), 0.04

232 (Cox-Snell), 0.05 (Nagelkerke). Model X²(2)=6.49, $\mathrm{p}=0.04$

\begin{tabular}{llcccc}
\hline & $\boldsymbol{\beta}(\mathrm{SE})$ & $\mathbf{p}$ & \multicolumn{3}{c}{$\mathbf{9 5 \%}$ CI for odds ratio } \\
\hline Constant & & & Lower & Odds Ratio & Upper \\
\hline Sex (Male) & $\begin{array}{l}0.68 \\
(0.24)\end{array}$ & 0.01 & & & \\
& $\begin{array}{l}\mathbf{- 0 . 7 1} \\
\mathbf{( 0 . 3 4 )}\end{array}$ & $\mathbf{0 . 0 3}$ & $\mathbf{0 . 2 5}$ & $\mathbf{0 . 4 9}$ & $\mathbf{0 . 9 4}$ \\
\hline $\begin{array}{l}\text { Rearing (Non- } \\
\text { mother) }\end{array}$ & $\begin{array}{l}0.48 \\
(0.35)\end{array}$ & 0.17 & 0.82 & 1.62 & 3.29 \\
\hline
\end{tabular}

The best-fit model for $\mathrm{ABN}-\mathrm{XC}$ included all three predictor variables and was statistically significant $\left(\mathrm{X}^{2}(5)=19.18, \mathrm{p}<0.01\right)$. Table 4 reports the regression coefficients and odds ratios for this model. The model explained $14.6 \%\left(\right.$ Nagelkerke $\left.\mathrm{R}^{2}\right)$ of the variance in $\mathrm{ABN}$ $\mathrm{XC}$ and correctly classified $64.2 \%$ of cases. Rearing had a positive relationship with $\mathrm{ABN}-\mathrm{XC}$ such that chimpanzees who were not mother-reared were 3.18 times more likely to exhibit ABN- 
$239 \mathrm{XC}$ than those who were mother-reared. The other variables included did not contribute

240 significantly to the model.

241 Table 4. Logistic regression model for non-coprophagy abnormal behavior (ABN-XC) with

242 predictor variables and constant. Significant variables are bolded. $\mathrm{R}^{2}=0.08$ (Hosmer-

243 Lemeshow), 0.11 (Cox-Snell), 0.15 (Nagelkerke). Model $X^{2}(5)=19.18, p<0.01$

\begin{tabular}{|c|c|c|c|c|c|}
\hline & \multirow[t]{2}{*}{$\beta(\mathrm{SE})$} & \multirow[t]{2}{*}{ p } & \multicolumn{3}{|c|}{ 95\% CI for odds ratio } \\
\hline & & & Lower & Odds Ratio & Upper \\
\hline Constant & $\begin{array}{l}-0.45 \\
(0.37)\end{array}$ & 0.22 & & & \\
\hline $\begin{array}{l}\text { Rearing (Non- } \\
\text { mother) }\end{array}$ & $\begin{array}{l}1.16 \\
(0.44)\end{array}$ & 0.01 & 1.37 & 3.18 & 7.65 \\
\hline Sex (Male) & $\begin{array}{l}-0.09 \\
(0.35)\end{array}$ & 0.80 & 0.46 & 0.92 & 1.81 \\
\hline Origin (Lab) & $\begin{array}{l}1.50 \\
(0.90)\end{array}$ & 0.09 & 0.89 & 4.50 & 34.5 \\
\hline Origin (Private) & $\begin{array}{l}-0.52 \\
(0.78)\end{array}$ & 0.50 & 0.13 & 0.60 & 2.76 \\
\hline Origin (Zoo) & $\begin{array}{l}-0.05 \\
(0.43)\end{array}$ & 0.91 & 0.41 & 0.95 & 2.26 \\
\hline
\end{tabular}

The best fit model for $\mathrm{ABN}-\mathrm{C}$ included all three predictor variables and was statistically significant, $X^{2}(5)=17.59, p<0.01$. The regression coefficients and odds ratios are reported in Table 5. The model explained 13.6\% (Nagelkerke's $\mathrm{R}^{2}$ ) of the variance in the exhibition of coprophagy and correctly classified $67.3 \%$ of cases. The variable sex was negatively related to coprophagy such that male chimpanzees were 3.57 times less likely to exhibit coprophagy than

250 female chimpanzees. The laboratory origin variable had a positive relationship with coprophagy

251 such that chimpanzees that were born in a laboratory were 5.33 times more likely to exhibit 252 coprophagy than those born in the wild. 
254 Table 5. Logistic regression model for coprophagy (ABN-C) with predictor variables and

255 constant. Significant variables are bolded. $\mathrm{R}^{2}=0.08$ (Hosmer-Lemeshow), 0.10 (Cox-Snell), 0.14

256 (Nagelkerke). Model $\mathrm{X}^{2}(5)=17.59, \mathrm{p}<0.01$

\begin{tabular}{lccccc}
\hline & $\boldsymbol{\beta}(\mathrm{SE})$ & $\mathbf{p}$ & \multicolumn{3}{c}{$\mathbf{9 5 \%}$ CI for odds ratio } \\
\hline & & & Lower & Odds Ratio & Upper \\
\hline Constant & -0.30 & 0.38 & & & \\
& $(0.38)$ & & & & \\
\hline Sex (Male) & $\mathbf{- 1 . 2 4}$ & $<\mathbf{0 . 0 1}$ & $\mathbf{0 . 1 4}$ & $\mathbf{0 . 2 9}$ & $\mathbf{0 . 5 9}$ \\
& $\mathbf{( 0 . 3 7 )}$ & & & & \\
\hline Origin (Lab) & $\mathbf{1 . 6 7}$ & $\mathbf{0 . 0 3}$ & $\mathbf{1 . 2 4}$ & $\mathbf{5 . 3 3}$ & $\mathbf{2 4 . 9 9}$ \\
& $\mathbf{( 0 . 7 6 )}$ & & & & \\
\hline Origin (Private) & 1.17 & 0.16 & 0.61 & 3.21 & 16.84 \\
& $(0.84)$ & & & & \\
\hline Origin (Zoo) & 0.63 & 0.16 & 0.79 & 1.87 & 4.63 \\
& $(0.45)$ & & & & \\
\hline Rearing (Non-mother) & -0.88 & 0.056 & 0.16 & 0.42 & 0.99 \\
& $(0.46)$ & & & & \\
\hline
\end{tabular}

257

258

Discussion

259

Our survey results revealed a lower prevalence of abnormal behavior in zoo-housed

chimpanzees compared to the most recent published evaluation (Birkett \& Newton-Fisher, 2011), which reported these behaviors as "endemic" and present in $100 \%$ of the zoo-housed subjects they sampled. Using similar categories of these behaviors in survey form, our data suggests that a lower prevalence of $64 \%$ of zoo-housed chimpanzees were observed to engage in some type of abnormal behavior over a two-year period. Although methodological differences may account for some of these differences, we assert that the current evaluation is a broader assessment of the prevalence of abnormal behavior in the zoo-housed chimpanzee population.

267 The current study draws from a substantially larger sample of zoos (26 institutions compared to 6) and subsequently surveys a broader range of individuals (165 subjects compared to 40 ). This 
269 is an important consideration given that, in this study and others, these behaviors are often linked

270 to early rearing histories, which should be adequately represented in the study sample.

One clear similarity in the results of these two assessments is the prevalence of

272

273

274

275

276

277

278

279

280

281

282

283

284

285

286

287

288

289

290

291 coprophagy as the most commonly reported abnormal behavior: $41 \%$ percent of the sample was reported to engage in this behavior. The link between this behavior and its utility as an indicator of wellbeing however, has recently been brought into question (Nash et al., 1999; Hopper, Freeman, \& Ross, 2016). There is growing evidence that coprophagy may be a socially-learned behavior and may not be as relevant an indicator of negative welfare as some other abnormal behaviors (Nash et al., 1999; Hook et al., 2002; Freeman \& Ross, 2014; Hopper, Freeman, \& Ross, 2016). Though a socially-learned behavior could still be an indicator of negative welfare, it can likely be distinguished from those behaviors that are more directly tied to environmental or social inadequacies. Our analysis of the variables that predict the occurrence of coprophagy in this sample support this concept in a number of ways. First, we found a significant sex difference in the prevalence of coprophagy such that female chimpanzees were 3.57 times more likely than male chimpanzees to exhibit this behavior. This finding mirrors a past assessment (Fritz et al., 1992) and may be linked to sex differences in social learning. A study by Lonsdorf (2005) has directly demonstrated the biased proclivity of female offspring to be the recipients of socially-transmitted tool-using behavior and we argue that coprophagy may be learned similarly through cultural transmission (Hopper, Freeman, \& Ross, 2016).

Another finding that would support the idea that coprophagy is indeed a socially-transmitted behavior is a link to rearing history. Indeed we found that mother-reared chimpanzees were 2.38 times more likely than non-mother-reared chimpanzees to exhibit coprophagy, though this finding did not reach statistical significance. Mother-reared chimpanzees are likely to have more 
292 exposure to other chimpanzees who display coprophagy than those chimpanzees raised in a

293 human setting (nursery or privately owned individuals typically live in smaller groups and their

294 companions are less likely to show coprophagy), so the opportunity to learn this behavior

295 socially may be heightened. In addition, mother-reared chimpanzees typically have more

296 developed social skills than those raised in nurseries or by humans (Spijkerman, 1997; Baker et

297 al., 2000; Kalcher-Sommersguter et al., 2011) which may allow them to better learn behaviors

298 from their mothers and others in their social groups. For these two reasons we would expect

299 these individuals to be more likely to learn a behavior like coprophagy. Also, given what we

300 know about the negative welfare outcomes for non-mother-reared chimpanzees (Fritz et al.,

301 1992; Nash et al., 1999; Martin, 2002; Kalcher-Sommersguter et al., 2011), if coprophagy was an

302 indicator of negative welfare, we would expect to see these chimpanzees exhibit more of this

303 behavior. Indeed the opposite trend is true in our sample, suggesting that coprophagy rates have

304 relatively little to do with welfare. These results reinforce the established relationship between

305 mother-rearing and elevated coprophagy (Nash et al., 1999; Bloomsmith et al., 2006; Hopper,

306 Freeman, \& Ross, 2016) and the idea that the link between welfare and this behavior should be

307 further evaluated.

308 Laboratory origin was also a significant predictor of coprophagy; chimpanzees born in research laboratory settings were 5.33 times more likely to exhibit coprophagy than chimpanzees

310 born in the wild. It is unclear why this relationship exists; further work is needed to compare the

311 effects of different physical and management environments on the behavior of captive

312 chimpanzees.

313 For some abnormal behaviors in primates we have empirical evidence to link the behaviors to 314 suboptimal environments such as social isolation and non-mother-rearing (rocking: Fritz et al., 
315 1992; self-injurious behavior: Harlow \& Harlow, 1965; Kraemer \& Clarke, 1990). These

316 behaviors were reported less in this chimpanzee sample; rocking and self-injurious behavior

317 were reported in fewer than $10 \%$ of chimpanzees. Overall, when we remove the occurrences of

318 coprophagy, the prevalence of abnormal behaviors in the sample decreases to about half of the

319 sample (48\%). The most prevalent behavior after coprophagy was hair plucking (32\%) which

320 has been recognized as a relatively common abnormal behavior in many primate species (Nash et

321 al., 1999; Lutz, Well, \& Novak, 2003; Less, Kuhar, \& Lukas, 2013; Brand \& Marchant, 2015).

322 However, the relationship between this behavior and psychological wellbeing is still unclear, as

323 heredity and social learning may influence the expression of hair plucking (Nash et al., 1999;

324 Hook et al., 2002; Less, Kuhar, \& Lukas, 2013). When examining the factors that influence the

325 expression of these abnormal behaviors, we find some substantive differences from those factors

326 influencing the expression of coprophagy.

As past studies have shown, rearing is associated with the occurrence of abnormal behaviors, although these studies have generally been correlational (Harlow \& Harlow, 1965; Rogers \& Davenport, 1969; Fritz et al., 1992; Nash et al., 1999; Martin, 2002). Our model supported this idea, demonstrating that chimpanzees who were not mother-reared were 3.18 times more likely to exhibit abnormal behavior (excluding coprophagy) than mother-reared chimpanzees. This rearing result is in the opposite direction as the trend revealed by our coprophagy-only model, again demonstrating differences in the ontogeny of these behaviors. We found no effect of sex, suggesting that in contrast to coprophagy, these behaviors are unlikely to be the result of social transmission and therefore may be more reliable indicators of individual welfare. The strong 336 effect of rearing also emphasizes that welfare evaluation must consider the contribution of 
337 historical variables to present behavior, rather than solely focusing on proximate factors as the

338 cause of all abnormal behavior.

339 Although this survey-based approach allows for a larger statistical sample, there are several

340 potential methodological weaknesses which should be considered. One limitation of a survey

341 method is that our results only show the prevalence of these behaviors without any information

342 about their frequency or duration. As such, individuals who perform these abnormal behaviors

343 on a daily basis cannot be distinguished from those who engage much more rarely, perhaps only

344 once over a two-year period. Furthermore, we are also unable to determine the potential effect of

345 other factors such as shifts in management protocols and social dynamics that may have

346 influenced the expression of abnormal behaviors over that time period. Though more intensive

347 observational studies could potentially address this weakness, they take considerably more time

348 and resources to conduct adequately.

349 Another possible weakness is that these findings are based on reports from animal

350 management staff working with the chimpanzees and may be vulnerable to subjective

351 interpretation or even diminished opportunity to observe these behaviors. Although these are

352 valid perspectives, we assert that even direct observations result in a relatively minute fraction of

353 a chimpanzee's daily activities and that more generalized observations taken over the course of

354 several years may be as likely to produce accurate prevalence estimates (Whitham \&

355 Wielebnowski, 2009; Less et al., 2012). Furthermore, the survey methods used here are

356 ultimately a conservative measure, as abnormal behaviors need only be observed once in a two

357 year period, and are therefore likely to be overestimating the prevalence of these behaviors. 
We assert that these survey data represent a useful evaluation of the prevalence of abnormal

359

360

361

362

363

364

365

behaviors in zoo-housed chimpanzees. This study provides a broad characterization of the occurrence of abnormal behavior in zoo-housed chimpanzees and elucidates some of the variables in the life histories of chimpanzees that contribute to these behaviors. When considering our results and the effects of rearing, sex, and origin on the occurrence of coprophagy compared to the other abnormal behaviors, it is apparent that coprophagy, despite its prevalence, is associated differently with these factors. This supports previous proposals for coprophagy to be classified separately when assessing abnormal behavior and welfare of chimpanzees (Hopper, Freeman, \& Ross, 2016). Overall, this study calls into question Birkett and Newton-Fisher's (2011) assertion that abnormal behavior is pervasive in zoo-housed chimpanzees, but we join those authors in their support for work to assess and ultimately improve captive environments for chimpanzees. We acknowledge that we were unable to assess all of the many factors potentially associated with abnormal behaviors, and as a result, we encourage future research to include information on factors such as genetic relatedness, age, social exposure and more detailed early historical variables in order to refine our knowledge of these behaviors. Understanding not only the prevalence of abnormal behaviors but also focusing efforts on determining the causes of those behaviors most likely to inform us about chimpanzees' psychological states, should be a priority for managers and welfare scientists.

\section{Acknowledgements}

We would like to thank all the zoo staff that completed surveys as part of this study. We would also like to thank the staff of the Fisher Center for their assistance in data analysis and manuscript preparation. 


\section{References}

402

403

404

405

406

407

408

409

410

411

412

413

414

415

416

417

418

419

420

421

422

423

AZA Ape TAG. 2010. Chimpanzee (Pan troglodytes) Care Manual. Association of Zoos and Aquariums, Silver Spring, MD.

Baker KC, Seres M, Aureli F, \& de Waal FBM. 2000. Injury risks among chimpanzees in three housing conditions. American Journal of Primatology, 51: 161-175.

Baker KC \& Easley SP. 1996. An analysis of regurgitation and reingestion in captive chimpanzees. Applied Animal Behaviour Science, 49: 403-415.

Bayne K \& Novak M. 1998. Behavioural disorders. In: B Bennett, C Abee, \& R Henrickson (Eds.), Nonhuman Primates in Biomedical Research: Diseases (pp. 485-500). New York: Academic Press.

Berkson G, Mason WA, \& Saxon SV. 1963. Situation and stimulus effects on stereotyped behaviors of chimpanzees. Journal of Comparative and Physiological Psychology, 56: 786-792.

Birkett LP \& Newton-Fisher NE. 2011. How Abnormal Is the Behaviour of Captive, Zoo-Living Chimpanzees? PLoS ONE 6(6): e20101. doi:10.1371/journal.pone.0020101

Bloomsmith MA, Baker KC, Ross SR, \& Lambeth SP. 2006. In: GP Sackett, G Ruppenthal, \& K Elias (Eds.), Nursery Rearing of Nonhuman Primates in the 21st Century. (pp. 289-312). New York: Kluver Academic Publishers.

Brand CM \& Marchant LF. 2015. Hair plucking in captive bonobos (Pan paniscus). Applied Animal Behaviour Science, 171: 192-196.

Brent L, Lee D, \& Eichberg J. 1989. The effects of single caging on chimpanzee behaviour. Laboratory Animal Science, 39, 345-346.

Erwin J \& Deni R. 1979. Strangers in a strange land: abnormal behaviors or abnormal environment? In: J Erwin, TL Maple \& G Mitchell (Eds.) Captivity and behavior, primates in breeding colonies, laboratories, and zoos. pp 1-28 New York: Van Nostrand Reinhold

Freeman HD. \& Ross SR. 2014. The impact of atypical early histories on pet or performer chimpanzees. PeerJ, 2: e579. https://doi.org/10.7717/peerj.579.

Feng X, Wang L, Yang S, Qin D, Wang J, Li C, Longbao L, Yuanye M, \& Hu X. 2012. Maternal separation produces lasting changes in cortisol and behavior in rhesus monkeys. Proceedings of the National Academy of Sciences, 108: 14312-14317.

Fritz J, Nash LT, Alford PL, \& Bowen JA. 1992. Abnormal behaviors, with a special focus on rocking, and reproductive competence in a large sample of captive chimpanzees (Pan troglodytes). American Journal of Primatology, 27: 161-176. 
424 Garner, JP. 2005. Stereotypies and other abnormal repetitive behaviors: Potential impact on

425 validity, reliability, and replicability of scientific outcomes. ILAR Journal, 46: 106-117.

426

427

428

429

430

431

432

433

434

435

436

437

438

439

440

441

442

443

444

445

446

447

448

449

450

451

452

453

454

455

456

457

458

459

460

461

462

463

464
Gottlieb DH, Capitanio JP and McCowan B. 2013. Risk factors for stereotypic behavior and selfbiting in rhesus macaques (Macaca mulatta): animal's history, current environment, and personality. American Journal of Primatology, 75: 995-1008.

Harlow HF \& Harlow MK. 1965. The effect of rearing conditions on behavior. International Journal of Psychiatry, 1: 43-51.

Hook MA, Lambeth SP, Perlman JE, Stavisky R, Bloomsmith MA, \& Schapiro, SJ. 2002. Inter-group variation in abnormal behavior in chimpanzees (Pan troglodytes) and rhesus macaques (Macaca mulatta). Applied Animal Behaviour Science, 76: 165-176.

Hopper LM, Freeman HD, \& Ross SR. 2016. Reconsidering coprophagy as an indicator of negative welfare for captive chimpanzees. Applied Animal Behaviour Science, 176: 112-119.

Hosey GR \& Skyner LJ. 2007. Self-injurious behavior in zoo primates. International Journal of Primatology, 28: 1431-1437.

Kalcher E, Franz C, Crailsheim K \& Preuschoft S. 2008. Differential onset of infantile deprivation produces distinctive long-term effects in adult ex-laboratory chimpanzees (Pan troglodytes). Developmental Psychobiology, 50: 777-788.

Kalcher-Sommersguter E, Preuschoft S, Crailscheim K, \& Franz C. 2011. Social competence of adult chimpanzees (Pan troglodytes) with severe deprivation history: I. an individual approach. Developmental Psychology, 47: 77-90.

Kraemer GW \& Clarke AS. 1990. The behavioral neurobiology of self-injurious behavior in rhesus monkeys. Progress in Neuro-Psychopharmacology and Biological Psychiatry, 14: S141S168.

Less EH, Kuhar CW, \& Lukas KE. 2013. Assessing the prevalence and characteristics of hairplucking behaviour in captive western lowland gorillas (Gorilla gorilla gorilla). Animal Welfare, 22: $175-183$.

Less EH, Kuhar CW, Dennis PM, \& Lukas KE. 2012. Assessing inactivity in zoo gorillas using keeper ratings and behavioral data. Applied Animal Behaviour Science, 137: 74-79.

Lonsdorf EV. 2005. Sex differences in the development of termite-fishing skills in the wild chimpanzees, Pan troglodytes schweinfurthii, of Gombe National Park, Tanzania. Animal Behaviour, 70: 673-683.

Lutz C, Well A and Novak M. 2003. Stereotypic and self-injurious behavior in rhesus macaques: a survey and retrospective analysis of environment and early experience. American Journal of Primatology, 60: 1-15. 
465

466

467

468

469

470

471

472

473

474

475

476

477

478

479

480

481

482

483

484

485

486

487

488

489

490

491

492

493

494

495

496

497

498

499

500

501

502

503

504

505

506

507

508

Martin JE. 2002. Early life experiences: Activity levels and abnormal behaviours in resocialised chimpanzees. Animal Welfare, 11: 419-436.

Mason GJ \& Latham NR. 2004. Can't stop, won't stop: is stereotypy a reliable animal welfare indicator? Animal Welfare, 13: 57-69.

Mason GJ. 1991. Stereotypies: a critical review. Animal Behaviour, 41: 1015-1037.

Nash LT, Fritz J, Alford PA, \& Brent L. 1999. Variables influencing the origins of diverse abnormal behaviors in a large sample of captive chimpanzees (Pan troglodytes). American Journal of Primatology, 48: 15-29.

Novak MA, Kinsey JH, Jorgensen MJ, \& Hazen TJ. 1998. Effects of puzzle feeders on pathological behaviour in individually housed rhesus monkeys. American Journal of Primatology, 28: 125-138.

Novak MA, Meyer JS, Lutz C, \& Tiefenbacher S. 2006. Deprived Environments: Developmental insights from primatology. In G. Mason \& J. Rushen (Eds.), Stereotypic animal behavior: Fundamentals and applications to welfare (pp. 153-189). UK: Cromwell Press.

Pfeiffer AJ \& Koebner LJ. 1978. The resocialization of single-caged chimpanzees and the establishment of an island colony. Journal of Medical Primatology, 7: 70-81.

PMCTrack. 2016. Population Management Center, Lincoln Park Zoo. Chicago.

R Core Team. 2015. R: A Language and Environment for Statistical Computing. Vienna, Austria. Retrieved from http://www.R-project.org

Rogers CM \& Davenport RK. 1969. Effects of restricted rearing on sexual behavior of chimpanzees. Developmental Psychology, 1: 200-204.

Ross SR. 2015. North American regional chimpanzee studbook (Pan troglodytes). Chicago: Lincoln Park Zoo.

Ross SR \& Bloomsmith MA. 2011. A comment on Birkett and Newton-Fisher (2011). In Reader Comments. PLOSOne.

Ross SR, Wagner KE, Schapiro SJ, Hau J, \& Lukas KE. 2011. Transfer and acclimatization effects on the behavior of two species of African great ape (Pan troglodytes and Gorilla gorilla gorilla) moved to a novel and naturalistic zoo environment. International Journal of Primatology, 32: 99-117.

Schapiro, SJ \& Bloomsmith MA. 1994. Behavioural effects of enrichment on singly-housed yearling rhesus monkeys. American Journal of Primatology, 32: 89-101. 
509 Spijkerman R, van Hooff J, Dienske H, \& Jens W. 1997. Differences in subadult behaviors of

510 chimpanzees living in peer groups and in a family group. International Journal of Primatology,

511 18: 439-454.

512 Symonds MRE \& Moussalli A. 2011. A brief guide to model selection, multimodel inference

513 and model averaging in behavioural ecology using Akaike's information criterion. Behavioral

514 Ecology and Sociobiology, 65: 13-21.

515 Vandeleest JJ, McCowan B \& Capitanio JP. 2011. Early rearing interacts with temperament and 516 housing to influence the risk for motor stereotypy in rhesus monkeys (Macaca mulatta). Applied 517 Animal Behavior Science, 132: 81-89.

518 Walsh S, Bramblett CA \& Alford PL. 1982. A vocabulary of abnormal behaviors in restrictively 519 reared chimpanzees. American Journal of Primatology, 3: 315-319.

520 Whitham JC \& Wielebnowski N. 2009. Animal-based welfare monitoring: using keeper ratings 521 as an assessment tool. Zoo Biology, 28: 545-560. 